Rev. Int. Contam. Ambie. 32 (3) 315-322, 2016

DOI: 10.20937/RICA.2016.32.03.06

\title{
EVALUACIÓN DE LA SUSTENTABILIDAD DE INSTALACIONES SOLARES CON COLECTORES DE PLACA PLANA Y TUBOS EVACUADOS
}

\author{
Enrique ALBIZZATI
}

Facultad de Ingeniería Química, Universidad Nacional del Litoral. Santiago del Estero 2654 Santa Fe, Argentina, 3000

Correo electrónico: albizati@fiq.unl.edu.ar

(Recibido marzo 2015; aceptado noviembre 2015)

Palabras clave: solar, calentamiento, agua, energía, emisiones

\section{RESUMEN}

La generación y el uso de los recursos energéticos producen efectos ambientales, de modo que en la evaluación de la aplicación de la energía solar es necesario considerar el ciclo completo de los aprovechamientos a través del análisis del ciclo de vida. En este trabajo se calculan indicadores de sustentabilidad para el calentamiento solar de agua, con electricidad (SE) y gas licuado (SGL) como energía auxiliar. Los dispositivos con colectores de placa plana (PP) tienen valores más bajos de la contribución solar a la demanda y la eficiencia térmica $(84.3 \%$ y $34 \%)$, mientras que con tubos evacuados (TE) ambos valores son $89.3 \%$ y $46.3 \%$ respectivamente. La energía solar térmica generada y las emisiones evitadas se determinan descontando sobre los valores brutos, el consumo de energía y las emisiones debido a los requerimientos específicos para la recogida de la radiación solar. La energía térmica total máxima generada es 189161.6 MJ (SGL-PP) y la mitigación máxima total es $21375.2 \mathrm{~kg} \mathrm{CO}_{2}$ eq (SE-PP). La energía térmica generada por año y por $\mathrm{m}^{2}$ de colector es más alta con gas licuado y el colector evacuado (SGL-TE). Sin embargo este tipo de colector maximiza las mitigaciones por año y por $\mathrm{m}^{2}$ usando electricidad (SE-TE). Los periodos de amortización energética de las instalaciones se encuentran entre 8.1 (SGL-TE) y 12.3 meses (SE-PP), mientras que los periodos de amortización ambientales entre 5.5 (SE-TE) y 12.1 (SGL-PP) meses.

Key words: solar, heating, water, energy, emissions.

\begin{abstract}
The generation and use of energy resources produce environmental effects, so that in the assessment of the application of solar energy is necessary to consider the complete cycle of the exploitation through the life cycle analysis. In this work sustainability indicators for solar installations of water heating, to use electricity (SE) and liquified gas (SLG) as auxiliary energy are calculated. Devices with flat plate collectors (FP) have lower values of solar contribution to the demand and thermal efficiency $(84.3 \%$ and $34.0 \%$ ) however, with evacuated tubes (ET) are $89.3 \%$ and $46.3 \%$, respectively. The solar thermal energy generated and the emissions avoided are determined by discounting on gross values of energy consumption and emissions due to the specific requirements for the collection of solar radiation. The maximum total thermal energy generated is $189161.6 \mathrm{MJ}$ (SLG-FP), and the maximum total mitigation is
\end{abstract}


$21375.2 \mathrm{~kg} \mathrm{CO}_{2}$ eq (SE-FP). The energy generated from the collector per year and per square meter is higher with liquefied gas and evacuated collector (SLG-ET). However this type of collector maximizes the mitigations per year and per square meter using electricity (SE-ET). The energy payback periods for these technologies are among 8.1 (SLG-ET) and 12.3 (SE-FP) months, but the environmental paybacks are among 5.5 (SE-ET) and 12.1 (SLG-FP) months.

\section{INTRODUCCIÓN}

Las predicciones realizadas sobre la futura disponibilidad de las energías no renovables y la contaminación ambiental provocada por los combustibles tradicionales (carbón, petróleo y gas natural) favorecen los proyectos que proponen la incorporación de fuentes energéticas renovables y no convencionales. El empleo intensivo de dichos combustibles fósiles en los países industrializados y la consiguiente emisión de gases de efecto invernadero, producen el denominado cambio climático global con su secuela de daños ambientales y socioeconómicos (IPCC 2013, OMM 2012).

La energía solar es uno de los recursos energéticos renovables más importantes con que se cuenta y existe un amplio y confiable conjunto de tecnologías para convertirla y utilizarla como energía térmica (Kalogirou 2009a, Albizzati 2012a, Duffie y Beckman 2013). En el año 2014, considerando sólo los equipos solares para calentamiento de agua, se contabilizaba una capacidad para producir $406 \mathrm{GW}$ térmicos en todo el mundo (REN21 2015).

Sin embargo, dados los impactos que provocan la generación y el uso de todas las fuentes energéticas, al evaluar la energía solar se debe analizar en forma completa el sistema de aprovechamiento aplicando el análisis de ciclo de vida (ACV). El ACV es un procedimiento que posibilita evaluar los impactos ambientales debidos a un producto o servicio. Al aplicar este método se deben contabilizar todas las entradas y salidas en todos los procesos que forman parte de su vida útil: desde la extracción de materias primas necesarias para su fabricación hasta su reciclaje y su disposición final (Consoli F. et al. 1993, Ihobe 2009).

En varios trabajos se aplicó el ACV con distintos objetivos y herramientas de cálculo, en los que se determinaron los consumos y beneficios tanto energéticos como ambientales, del calentamiento solar de agua doméstica (Ardente et al. 2005, ReyMartínez et al. 2008, Kalogirou 2009b, Hang et al. 2012, Koroneos y Nanaki 2012, Greening y Azapagic 2014, Lamnatou et al. 2014). Un equipamiento usado comúnmente para calentar agua en viviendas unifamiliares está compuesto por los colectores solares, el tanque de almacenamiento y las cañerías de distribución del fluido, además de una fuente energética auxiliar.

En este trabajo se calcula en primer lugar el aporte solar y la eficiencia térmica mensual y anual de sistemas solares combinados con electricidad y gas licuado como energías auxiliares. Luego para cada sistema se determinan la energía solar térmica generada y la emisión de gases evitadas al operar con energía solar. A los valores originados por el reemplazo de electricidad y gas licuado, se descuentan la energía consumida y la emisión provocada por los requerimientos propios de la instalación del sistema de captación solar. Finalmente, se estiman los periodos de amortización energética necesarios para recuperar la energía consumida y ambiental que generan los gases emitidos por cada instalación.

\section{Instalaciones y aporte solar a la demanda térmica}

El fluido circula por convección natural entre los colectores y el tanque acumulador. Los colectores solares están posicionados en Santa Fe (Argentina), con $30^{\circ}$ de inclinación al norte geográfico (Fig. 1).

El consumo de agua doméstico se fija en $240 \mathrm{~L}$ diarios a $45^{\circ} \mathrm{C}$, siendo la temperatura del agua fría $18{ }^{\circ} \mathrm{C}$ durante todo el año. Como se observa en el cuadro I, son cuatro las instalaciones estudiadas, dos solar-electricidad (SE) y dos solar-gas licuado (SGL), con colectores de placa plana (PP) y de tubos evacuados (TE). Se estima que las instalaciones pueden satisfacer la demanda de una familia de cinco personas y sus características técnicas responden a la

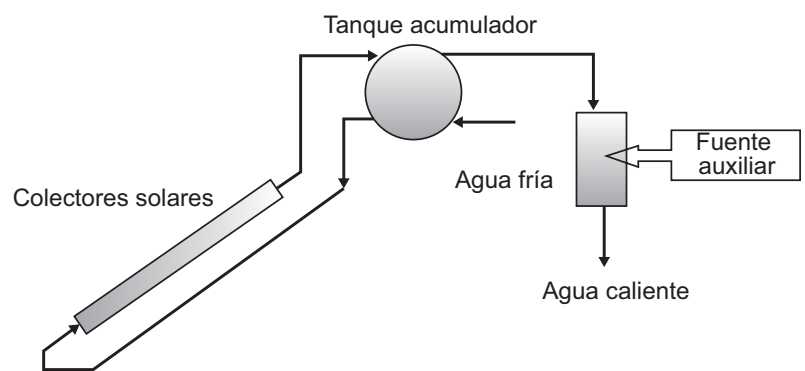

Fig. 1. Esquema de la instalación combinada para calentamiento de agua 
CUADRO I. INSTALACIONES COMBINADAS PARA CALENTAMIENTO DE AGUA

\begin{tabular}{lccccc}
\hline Instalación & $\begin{array}{c}\mathrm{T} \\
(\mathrm{L})\end{array}$ & $\begin{array}{c}\mathrm{A} \\
\left(\mathrm{m}^{2}\right)\end{array}$ & $\begin{array}{c}\mathrm{B} \\
\left({ }^{\circ}\right)\end{array}$ & $\begin{array}{c}\mathrm{F}_{\mathrm{R}} \mathrm{E} \\
(-)\end{array}$ & $\begin{array}{c}\mathrm{F}_{\mathrm{R}} \mathrm{U}_{\mathrm{C}} \\
\left(\mathrm{W} / \mathrm{m}^{2}{ }^{\circ} \mathrm{C}\right)\end{array}$ \\
\hline SE-PP & 240 & 3.6 & 30 & 0.7 & 6.0 \\
SE-TE & 200 & 2.8 & 30 & 0.7 & 2.0 \\
SGL-PP & 240 & 3.6 & 30 & 0.7 & 6.0 \\
SGL-TE & 200 & 2.8 & 30 & 0.7 & 2.0 \\
\hline
\end{tabular}

SE-PP (solar-electricidad, colectores de placa plana) SE-TE (solarelectricidad, colector de tubos evacuados) SGL-PP (solar-gas licuado, colectores de placa plana) SGL-TE (solar-gas licuado, colector de tubos evacuados), T: volumen de agua en el tanque de almacenamiento, A: área total de captación solar, B: ángulo de inclinación de los colectores solares, $\mathrm{F}_{\mathrm{R}} \mathrm{E}$ : producto del factor de remoción del calor por la eficiencia óptica de los colectores solares, $F_{R} U_{c}$ : producto del factor de remoción por el coeficiente total de pérdidas de calor de los colectores solares

oferta comercial existente y a las recomendaciones realizadas por los vendedores a los usuarios.

Dos de las instalaciones combinadas para el calentamiento poseen dos colectores solares de placa plana de $1.8 \mathrm{~m}^{2}$ cada uno, el tanque acumulador es de 240 L y su vida útil de 20 años. En las otras, el colector solar es de tubos evacuados de $2.8 \mathrm{~m}^{2}$, posee un tanque de 200 L y 15 años de utilidad.

Cada una de las instalaciones se caracteriza por el volumen de agua en el tanque (T), el área de colección (A), la inclinación de los colectores respecto al plano horizontal (B) y los parámetros propios de éstos. Dichos parámetros $\left(\mathrm{F}_{\mathrm{R}} \mathrm{E}\right.$ y $\left.\mathrm{F}_{\mathrm{R}} \mathrm{U}_{\mathrm{C}}\right)$ están asociados a $\mathrm{E}$, la eficiencia óptica, $\mathrm{F}_{\mathrm{R}}$ el factor de remoción del calor y $\mathrm{U}_{\mathrm{C}}$ el coeficiente total de pérdidas de calor, los que se determinan conforme a las normas vigentes (ANSI/ASHRAE 93 2010).

En los colectores solares planos adoptados la radiación se transmite por una cubierta de vidrio, es recibida y absorbida por una placa de acero inoxidable que la transforma en energía térmica. Esta energía se transfiere por conducción en la placa y se incorpora al fluido. El agua circula por canales conformados entre la placa superior e inferior, aumentando su temperatura. Entre la placa y la cubierta hay una cámara de aire. Se utiliza un material aislante en el fondo y en los laterales. Se coloca una caja de chapa galvanizada que contiene todos los elementos que conforman el colector.

En los otros colectores solares el agua circula por un conjunto de tubos evacuados dispuestos en paralelo. Se propone para cada tubo dos conductos concéntricos de vidrio borosilicato separados por una cámara al vacío. La radiación se transmite en el tubo exterior y se absorbe en el tubo interior, el que posee un recubrimiento superficial especialmente desarrollado para tal efecto. El fluido circula en U, entrando y saliendo del tanque acumulador por el extremo superior del tubo interno. Ambos tubos están cerrados por fusión en el extremo inferior y el colector tiene además una chapa reflectora debajo del conjunto de tubos de vidrio evacuados.

Para calcular la radiación solar diaria promedio recibida por los colectores se necesitan conocer los valores de los componentes de la luz directa y difusa de la radiación en el plano horizontal. La radiación se contabiliza como energía por unidad de tiempo y por unidad de área de colección. La radiación solar global promedio diario mensual $(\mathrm{H})$, es la suma de la radiación directa del $\mathrm{sol}\left(\mathrm{H}_{\mathrm{D}}\right)$ y la radiación difusa del cielo $\left(\mathrm{H}_{\mathrm{d}}\right)$ :

$\mathrm{H}=\mathrm{H}_{\mathrm{D}}+\mathrm{H}_{\mathrm{d}}$

Las componentes de la luz directa y difusa de la radiación solar horizontal en cada mes del año se obtienen para la ciudad de Santa Fe de la base de datos del Centro de Datos de Ciencias Atmosféricas (NASA 2014). La radiación solar total que reciben los colectores $\left(\mathrm{H}_{\mathrm{T}}\right)$ es la suma de la radiación directa, la radiación difusa recibida desde el cielo y la radiación difusa reflejada por el suelo.

La radiación solar promedio diaria que incide sobre los colectores se obtiene por el modelo propuesto por Liu-Jordan y extendido por Klein. Luego, $\mathrm{H}_{\mathrm{T}}$ es el producto de la radiación global horizontal multiplicada por un factor $\mathrm{R}$, que indica la variación de la radiación sobre los colectores respecto a la que incide en el plano horizontal (Duffie y Beckman 2013):

$\mathrm{H}_{\mathrm{T}}=\mathrm{R} \mathrm{H}$

El cuadro II presenta los valores de la radiación solar global promedio mensual en plano horizontal y los resultantes del cálculo sobre los colectores solares posicionados a $30^{\circ}$ al norte geográfico.

La demanda de energía térmica mensual (Q) en las instalaciones se calcula con el volumen de agua consumido (V), la densidad del agua (p), el calor específico del agua (c), la temperatura del agua caliente $\left(\mathrm{T}_{\mathrm{c}}\right.$ ) y la temperatura del agua fría $\left(\mathrm{T}_{\mathrm{f}}\right)$ :

$\mathrm{Q}=\mathrm{Vpc}\left(\mathrm{T}_{\mathrm{c}}-\mathrm{T}_{\mathrm{f}}\right)$

El aporte de la energía solar a la demanda en las instalaciones combinadas se determina con el método de la carta F (Duffie y Beckman 2013). En este método la fracción de la energía solar aportada en cada mes (f), se determina con: 
CUADRO II. RADIACIÓN SOLAR GLOBAL HORIZONTAL Y TOTAL MENSUAL SOBRE LOS COLECTORES SOLARES

\begin{tabular}{|c|c|c|c|c|c|c|c|c|c|c|c|c|}
\hline MES & 1 & 2 & 3 & 4 & 5 & 6 & 7 & 8 & 9 & 10 & 11 & 12 \\
\hline $\begin{array}{l}\mathrm{H} \\
\left(\mathrm{MJ} / \mathrm{m}^{2}\right) \\
\mathrm{H}_{\mathrm{T}}\end{array}$ & 24.70 & 21.82 & 18.14 & 13.61 & 10.84 & 8.89 & 10.15 & 13.39 & 17.50 & 20.30 & 23.76 & 25.02 \\
\hline$\left(\mathrm{MJ} / \mathrm{m}^{2}\right)$ & 22.11 & 21.05 & 19.49 & 16.66 & 15.26 & 13.23 & 14.92 & 17.70 & 19.99 & 20.25 & 21.68 & 21.97 \\
\hline
\end{tabular}

$\mathrm{H}$ (radiación solar global sobre plano horizontal) $\mathrm{H}_{\mathrm{T}}$ (radiación solar total sobre plano inclinado de captación)

$\mathrm{f}=1.029 \mathrm{Y}-0.065 \mathrm{X}-0.245 \mathrm{Y}^{2}$

$+0.0018 \mathrm{X}^{2}+0.0215 \mathrm{Y}^{3}$

En la expresión (4), X relaciona la cantidad de radiación solar incidente sobre los colectores con la demanda térmica, mientras que $\mathrm{Y}$ es el cociente entre las pérdidas de energía del sistema y la citada demanda. Estos parámetros se corrigen considerando los valores de la temperatura del agua caliente, el volumen de agua en el tanque, la eficiencia óptica y el factor de remoción de calor del colector solar.

La fracción anual aportada por la energía solar a la demanda térmica $(\mathrm{F})$ resulta de una sumatoria aplicada para los doce meses del año:

$\mathrm{F}=\Sigma(\mathrm{f} \mathrm{Q})_{\mathrm{i}} / \Sigma \mathrm{Q}_{\mathrm{i}}$

A su vez, la eficiencia térmica anual de cada instalación solar $\left(\mathrm{E}_{\mathrm{t}}\right)$ se calcula como el cociente entre la energía solar aportada y la radiación total solar sobre los colectores en los " $n$ " días de cada mes:

$\mathrm{E}_{\mathrm{t}}=\Sigma(\mathrm{fQ})_{\mathrm{i}} / \mathrm{A} \Sigma\left(\mathrm{H}_{\mathrm{T}} \mathrm{n}\right)_{\mathrm{i}}$

Los resultados obtenidos mediante la aplicación del método de la carta F se muestran en el cuadro III, mientras que en el cuadro IV se visualizan los aportes solares y las eficiencias térmicas anuales que se han analizado para las instalaciones. Las diferencias que se verifican en dichos cuadros se deben a que los colectores de placa plana tienen menor aporte solar y eficiencia térmica que los de tubos evacuados en los meses más fríos, debido a que es mayor el coeficiente de pérdidas de calor.

CUADRO IV. RADIACIÓN INCIDENTE, APORTE SOLAR Y EFICIENCIA TÉRMICAANUAL DE LAS INSTALACIONES

\begin{tabular}{lccc}
\hline Instalación & $\begin{array}{c}\text { Radiación } \\
\text { anual (MJ) }\end{array}$ & $\begin{array}{c}\text { Aporte } \\
\text { solar (\%) }\end{array}$ & $\begin{array}{c}\text { Eficiencia } \\
\text { térmica (\%) }\end{array}$ \\
\hline $\begin{array}{l}\text { SE-PP } \\
\text { SGL-PP }\end{array}$ & 24549.7 & 84.3 & 34.0 \\
$\begin{array}{l}\text { SE-TE } \\
\text { SGL-TE }\end{array}$ & 19094.2 & 89.3 & 46.3 \\
\hline
\end{tabular}

SE-PP (solar-electricidad, colectores de placa plana) SE-TE (solar-electricidad, colector de tubos evacuados) SGL-PP (solar-gas licuado, colectores de placa plana) SGL-TE (solar-gas licuado, colector de tubos evacuados)

\section{Análisis de ciclo de vida en las instalaciones}

En el ACV de las instalaciones solares se deben contemplar aquellas actividades que se llevan a cabo y que tienen la relevancia suficiente (Fig. 2). El inventario del ciclo de vida o relevamiento de las entradas y salidas sienta las bases para evaluar

CUADRO III. APLICACIÓN MES A MES DEL MÉTODO DE LA CARTA F EN LAS INSTALACIONES

\begin{tabular}{|c|c|c|c|c|c|c|c|c|c|c|c|c|c|}
\hline Instalación & Mes & 1 & 2 & 3 & 4 & 5 & 6 & 7 & 8 & 9 & 10 & 11 & 12 \\
\hline \multirow{3}{*}{$\begin{array}{l}\text { SE-PP } \\
\text { SGL-PP }\end{array}$} & $X$ & 4.78 & 4.84 & 5.08 & 5.66 & 6.15 & 6.54 & 6.69 & 6.28 & 5.94 & 5.44 & 5.13 & 4.65 \\
\hline & $\mathrm{Y}$ & 2.05 & 1.96 & 1.81 & 1.55 & 1.42 & 1.23 & 1.34 & 1.64 & 1.86 & 1.88 & 2.01 & 2.04 \\
\hline & $\mathrm{f}$ & 1.00 & 0.96 & 0.90 & 0.78 & 0.70 & 0.59 & 0.66 & 0.79 & 0.88 & 0.91 & 0.97 & 0.99 \\
\hline \multirow{3}{*}{$\begin{array}{l}\text { SE-TE } \\
\text { SGL-TE }\end{array}$} & $X$ & 1.18 & 1.23 & 1.29 & 1.44 & 1.57 & 1.67 & 1.70 & 1.60 & 1.51 & 1.39 & 1.31 & 1.22 \\
\hline & $\mathrm{Y}$ & 1.60 & 1.52 & 1.41 & 1.20 & 1.10 & 0.96 & 1.08 & 1.28 & 1.44 & 1.46 & 1.57 & 1.59 \\
\hline & $\mathrm{f}$ & 1.00 & 1.00 & 0.94 & 0.83 & 0.77 & 0.68 & 0.75 & 0.86 & 0.95 & 0.96 & 1.00 & 1.00 \\
\hline
\end{tabular}

SE-PP (solar-electricidad, colectores de placa plana) SE-TE (solar-electricidad, colector de tubos evacuados) SGL-PP (solar-gas licuado, colectores de placa plana) SGL-TE (solar-gas licuado, colector de tubos evacuados), $\mathrm{X}$ : parámetro relacionado con las pérdidas térmicas de los colectores solares, Y: parámetro que depende de la energía solar absorbida de los colectores solares, f: fracción mensual de la demanda térmica que es cubierta con energía solar 


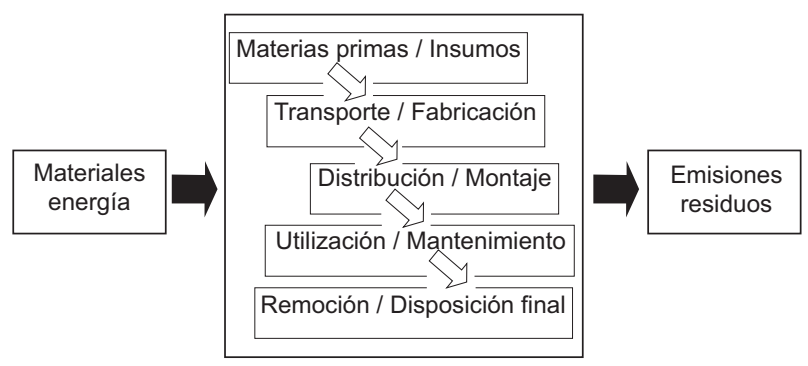

Fig. 2. Esquema del ciclo de vida de la instalación solar

los impactos ambientales. En dicho inventario se utilizan dos categorías: el consumo energético (la energía consumida en la obtención de las materias primas, fabricación, distribución, uso y fin de vida del producto) y el cambio climático (medida de la temperatura que muestra en promedio un aumento en la temperatura de la atmósfera terrestre y de los océanos en las últimas décadas). En el calentamiento global se considera el impacto de todos los gases de efecto invernadero en equivalentes de dióxido de carbono.

En cada instalación combinada de calentamiento solar de agua la generación de energía térmica y la mitigación de la emisión de gases se calculan comparando cada instalación con otra que usa solamente electricidad o gas licuado. Los valores brutos de la generación térmica y de la mitigación que se deben al aporte solar durante el funcionamiento de la instalación combinada se obtienen con:

$\mathrm{E}_{\mathrm{b}}=\Sigma \mathrm{Q}_{\mathrm{i}} \mathrm{NF} \mathrm{c}_{\mathrm{a}}$

En la ecuación anterior, $\Sigma Q_{i}$ es la demanda térmica anual, $\mathrm{N}$ son los años de vida útil de la instalación y $\mathrm{F}$ es la fracción de la demanda térmica cubierta con energía solar. Respecto a $c_{a}$, al calcular la energía térmica generada vale uno y es diferente al determinar la mitigación de la emisión según la fuente de energía auxiliar utilizada.

Para calcular $\mathrm{c}_{\mathrm{a}}$ se emplea información de la emisiones debidas a los combustibles fósiles y que está relacionada con el tipo de generación eléctrica en Argentina (Consejo Mundial de Energía 2004). Las emisiones de gases en la conversión a energía térmica son $0.125 \mathrm{~kg} \mathrm{CO}_{2} \mathrm{eq} / \mathrm{MJ}(0.450 \mathrm{~kg} \mathrm{CO}$ eq $/ \mathrm{kW} \mathrm{h})$ y $0.083 \mathrm{~kg} \mathrm{CO}_{2}$ eq $/ \mathrm{MJ}\left(0.299 \mathrm{~kg} \mathrm{CO}_{2}\right.$ eq $\left./ \mathrm{kW} \mathrm{h}\right)$ para la energía eléctrica y el gas licuado respectivamente. Estos valores se corrigen considerando una eficiencia en la conversión del $95 \%$ cuando se consume electricidad y del $85 \%$ si el combustible es gas licuado.

Para determinar luego la generación de energía térmica y la mitigación de emisiones netas en cada instalación combinada se restan a los valores brutos, los efectos debidos a los consumos energéticos y a las emisiones de gases en el ciclo de vida correspondientes a la instalación solar y a su funcionamiento (Albizzati 2012b). Entonces dichos valores netos se calculan con:

$E_{n}=E_{b}-e_{i} A$

En cada situación, $e_{i}$ representa la energía consumida o los gases emitidos por unidad de área de colección. En su cálculo se suman las contribuciones asociadas con los colectores solares $\left(\mathrm{e}_{\mathrm{c}}\right)$, con el tanque de almacenamiento $\left(\mathrm{e}_{\mathrm{t}}\right)$, con otros materiales como cañerías, aislantes, soportes, pinturas, selladores y uniones $\left(\mathrm{e}_{\mathrm{m}}\right)$ y con actividades complementarias como transporte, fabricación, montaje y mantenimiento $\left(\mathrm{e}_{\mathrm{f}}\right)$ del dispositivo solar:

$\mathrm{e}_{\mathrm{i}}=\mathrm{e}_{\mathrm{c}}+\mathrm{e}_{\mathrm{t}}+\mathrm{e}_{\mathrm{m}}+\mathrm{e}_{\mathrm{f}}$

Para encontrar el valor de $\mathrm{e}_{\mathrm{i}}$ de la instalación solar se usan datos de la energía consumida y de las emisiones de los materiales involucrados en cada una de ellas (Alcorn 2003, Hammond y Jones 2011).

Finalmente, como se sustituyen fuentes tradicionales por energía solar, es apropiado calcular los periodos de amortización energética y ambiental de las instalaciones. El periodo de amortización energética puede definirse como el tiempo de funcionamiento necesario para recuperar la energía consumida en el ciclo de vida de cada instalación, mientras que el periodo de amortización ambiental se considera en forma semejante, pero incorporando la cantidad de gases de efecto invernadero.

Los periodos de amortización energética y ambiental se determinan en meses con la expresión:

$\mathrm{P}_{\mathrm{a}}=12 \mathrm{e}_{\mathrm{i}} \mathrm{A} / \Sigma \mathrm{Q}_{\mathrm{i}} \mathrm{c}_{\mathrm{a}}(2 \mathrm{~F}-1)$

\section{Resultados obtenidos y discusión}

Los valores brutos de la generación de energía y la mitigación de emisiones se deben al aporte solar durante la operación de cada sistema y al obtener los valores netos, éstos se descuentan a los valores brutos calculados, propios a cada una de las instalaciones de captación solar adoptadas.

Para determinar los valores que se muestran en el cuadro $\mathbf{V}$, se siguen los métodos aplicados en un trabajo anterior (Albizzati 2012b). Al calcular el consumo y las emisiones de las instalaciones que usan colectores planos, pinturas, recubrimientos, 
CUADRO V. CONSUMO Y EMISIONES PROPIOS DE LAS INSTALACIONES DE CAPTACIÓN SOLAR

\begin{tabular}{|c|c|c|c|c|c|c|c|c|}
\hline \multirow{3}{*}{ Materiales e insumos } & \multicolumn{4}{|c|}{ Colectores placa plana } & \multicolumn{4}{|c|}{ Colector tubos evacuados } \\
\hline & \multicolumn{2}{|c|}{ Consumo } & \multicolumn{2}{|c|}{ Emisión } & \multicolumn{2}{|c|}{ Consumo } & \multicolumn{2}{|c|}{ Emisión } \\
\hline & MJ & $\%$ & $\mathrm{~kg} \mathrm{CO} 2$ eq & $\%$ & MJ & $\%$ & $\mathrm{~kg} \mathrm{CO}_{2}$ eq & $\%$ \\
\hline Colectores solares & 3547.7 & 48.2 & 343.0 & 50.9 & 2525.7 & 41.0 & 179.9 & 38.0 \\
\hline Tanque de almacenamiento & 1117.4 & 15.2 & 110.8 & 16.4 & 931.3 & 15.1 & 92.3 & 19.5 \\
\hline Materiales varios & 2024.7 & 27.5 & 159.3 & 23.6 & 2030.3 & 32.9 & 150.1 & 31.7 \\
\hline Actividades complementarias & 669.0 & 9.1 & 61.3 & 9.1 & 675.0 & 11.0 & 51.2 & 10.8 \\
\hline Total & 7358.8 & 100 & 674.4 & 100 & 6162.2 & 100 & 473.5 & 100 \\
\hline
\end{tabular}

selladores y uniones, se obtiene que representan el $10 \%$ de la suma de los debidos a los colectores y el tanque. Adicionalmente, para los mismos sistemas, las actividades complementarias representan un $10 \%$ del consumo y las emisiones de todos los elementos que se tienen en cuenta al valorar las instalaciones. En lo relativo al colector de tubos evacuados, ambos porcentajes aumentan al $15 \%$ en lo que respecta a los colectores solares y los restantes se mantienen igual al caso anterior.

Se observa también en el cuadro $\mathbf{V}$ que en las instalaciones SE-PP y SGL-PP los colectores representan el $48.2 \%$ del consumo de energía y el $50.9 \%$ de la emisión de gases, mientras que $7358.8 \mathrm{MJ}$ es el consumo y $674.4 \mathrm{~kg} \mathrm{CO}_{2}$ eq es la emisión. Lo anterior al considerar los colectores solares, el tanque de almacenamiento, los materiales varios y las actividades complementarias. En los equipos con tubos evacuados SE-TE y SGL-TE, el colector cubre el $41.0 \%$ del consumo y el $38.0 \%$ de la emisión, el consumo total es $6162.2 \mathrm{MJ}$ y la emisión total es $473.5 \mathrm{~kg} \mathrm{CO} 2$ eq.

Los cálculos realizados de la generación y la mitigación netas se ven en el cuadro VI. Resulta que la máxima energía generada al operar con energía solar

CUADRO VI. ENERGÍA TÉRMICA GENERADA Y MITIGACIÓN DE EMISIONES NETAS TOTALES EN LA VIDA ÚTIL

\begin{tabular}{lccc}
\hline Instalación & $\begin{array}{c}\text { Generación } \\
\mathrm{MJ}\end{array}$ & $\begin{array}{c}\text { Mitigación } \\
\mathrm{kg} \mathrm{CO} \text { eq }\end{array}$ & $\begin{array}{c}\text { Mitigación/ } \\
\text { generación } \\
\mathrm{kg} \mathrm{CO}_{2} \text { eq/MJ }\end{array}$ \\
\hline SE-PP & 168536.8 & 21375.2 & 0.127 \\
SE-TE & 133520.2 & 17036.5 & 0.128 \\
SGL-PP & 189161.6 & 15695.8 & 0.083 \\
SGL-TE & 149898.8 & 12526.3 & 0.084 \\
\hline
\end{tabular}

SE-PP (solar-electricidad, colectores de placa plana) SE-TE (solar-electricidad, colector de tubos evacuados) SGL-PP (solar-gas licuado, colectores de placa plana) SGL-TE (solar-gas licuado, colector de tubos evacuados) es 189161.6 MJ (SGL-PP) y la mitigación máxima es $21375.2 \mathrm{~kg} \mathrm{C02}$ eq (SE-PP). Cuando se comparan dos instalaciones que usan los mismos colectores se logra una mayor generación de energía térmica con gas licuado, es decir que es mayor el beneficio energético. Sin embargo es superior el beneficio ambiental con electricidad, ya que se obtiene una mayor mitigación.

Como se observa en el cuadro VII, las energías generadas por año y por $\mathrm{m}^{2}$ de captación solar son mayores al usar gas licuado y el colector evacuado (SGL-TE), pero este tipo de colector maximiza las mitigaciones por año y por $\mathrm{m}^{2}$ si se utiliza electricidad como fuente energética auxiliar (SE-TE).

Los periodos de amortización energética resultantes están entre 8.1 (SGL-TE) y 12.3 (SE-PP) meses y los de amortización ambiental entre 5.5 (SE-TE) y 12.1 (SGL-PP) meses (Fig. 3). El cociente entre los periodos de amortización ambiental y energética es menor que 1.0, para las instalaciones SE-PP (0.732), SE-TE (0.611) y SGL-TE (0.914). En cambio cuando se usan colectores solares planos y gas licuado (SGL-PP) dicho cociente es 1.100 .

CUADRO VII. ENERGÍA TÉRMICA GENERADA Y MITIGACIÓN DE EMISIONES NETAS POR AÑO Y POR METRO CUADRADO DE COLECTOR

\begin{tabular}{lccccc}
\hline \multirow{2}{*}{ Instalación } & \multicolumn{2}{c}{ Generación } & & \multicolumn{2}{c}{ Mitigación } \\
\cline { 2 - 3 } \cline { 5 - 6 } & $\mathrm{MJ} / \mathrm{año}$ & $\mathrm{MJ} / \mathrm{m}^{2}$ & & $\begin{array}{c}\mathrm{kg} \mathrm{CO} \\
\mathrm{eq} / \mathrm{año}\end{array}$ & $\begin{array}{c}\mathrm{kg} \mathrm{CO} \\
\mathrm{eq} / \mathrm{m}^{2}\end{array}$ \\
\hline SE-PP & 8426.8 & 46815.8 & & 1068.8 & 5937.6 \\
SE-TE & 8901.3 & 47685.8 & & 1135.8 & 6084.5 \\
SGL-PP & 9458.1 & 52544.9 & & 784.8 & 4359.9 \\
SGL-TE & 9993.3 & 53535.3 & & 835.1 & 4473.7 \\
\hline
\end{tabular}

SE-PP (solar-electricidad, colectores de placa plana) SE-TE (solar-electricidad, colector de tubos evacuados) SGL-PP (solar-gas licuado, colectores de placa plana) SGL-TE (solar-gas licuado, colector de tubos evacuados) 


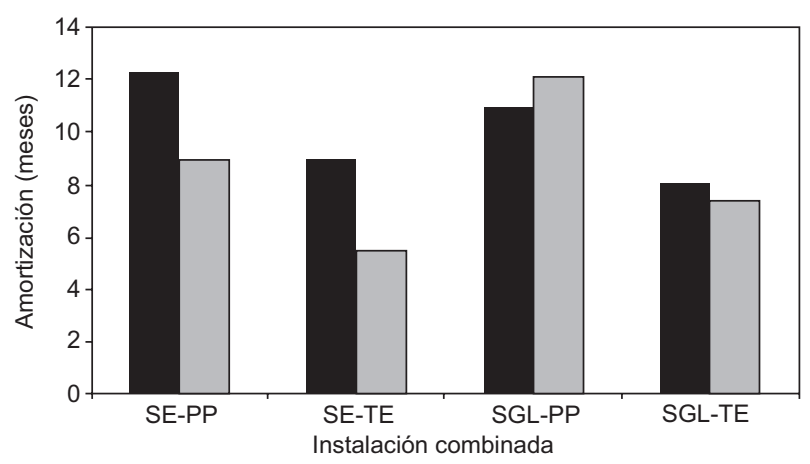

Fig. 3. Periodos de amortización energética ( ) y ambiental ( $\square)$ de las instalaciones. SE-PP(solar-electricidad, colectores de placa plana) SE-TE (solar-electricidad, colector de tubos evacuados) SGL-PP (solar-gas licuado, colectores de placa plana) SGL-TE (solar-gas licuado, colector de tubos evacuados)

\section{CONCLUSIONES}

Con el ACV se han calculado indicadores para establecer el impacto energético y ambiental del empleo de energía solar en instalaciones de calentamiento de agua que utilizan electricidad y gas licuado como fuente auxiliar. En los sistemas de captación solar con colectores planos los requerimientos propios de éstos representan el $48.2 \%$ del consumo de energía y el $50.9 \%$ de las emisiones de gases. Mientras que en los equipos con el colector de tubos evacuados los respectivos porcentajes bajan a $41.0 \%$ y $38.0 \%$.

La máxima energía total neta generada se alcanza en instalaciones con colectores planos y gas licuado (SGL-PP), pero la mitigación neta de las emisiones de gases es máxima con los mismos colectores combinados con electricidad (SE-PP). Debe tomarse en cuenta que, para la electricidad, aquí se han considerado las fuentes de energía primaria de Argentina y que si fueran diferentes, éstas podrían cambiar el impacto ambiental asociado con dicha fuente auxiliar.

Como resultado de los cálculos se pudo verificar que en instalaciones que tienen el mismo tipo de colector solar, siempre es mayor la generación de energía neta funcionando con gas licuado, pero es mayor la mitigación neta con energía eléctrica auxiliar. La utilización de gas licuado y el colector evacuado (SGL-TE) maximiza las energías generadas por año y por $\mathrm{m}^{2}$. Con el mismo colector son máximas las mitigaciones por año y por $\mathrm{m}^{2}$ cuando se emplea electricidad (SE-TE). Los periodos de amortización energética mínimo y máximo son 8.1 (SGL-TE) y 12.3 (SE-PP) meses, mientras que los correspondientes a la amortización ambiental son 5.5 (SE-TE) y 12.1 (SGL-PP) meses.

\section{AGRADECIMIENTOS}

Se agradece a la Facultad de Ingeniería Química de la Universidad Nacional del Litoral la colaboración recibida para la realización de este trabajo.

\section{REFERENCIAS}

Albizzati E. (2012a). Energía solar térmica: principios básicos y aplicaciones tecnológicas. Centro de Publicaciones de la Universidad Nacional del Litoral, Santa $\mathrm{Fe}$, Argentina, $150 \mathrm{pp}$.

Albizzati E. (2012b). Cálculos de la energía generada y la mitigación de emisiones de gases en instalaciones para calentamiento solar de agua. Energías Renovables y Medio Ambiente 31, 9-16.

Alcorn A. (2003). Embodied energy and $\mathrm{CO}_{2}$ coefficients for NZ buildings materials. Centre for Building Performance Research. Victoria University of Wellington, Nueva Zelanda, $31 \mathrm{pp}$.

Ardente F., Beccali G., Cellura M. y Lo Brano V. (2005). Life cycle assessment of a solar thermal collector. Renew. Energ. 30, 1031-1054.

DOI: $10.1016 /$ j.renene.2004.09.009.

ANSI/ASHRAE 93 (2010). Methods of testing to determine the thermal performance of solar collectors. American National Standards Institute/American Society of Heating, Refrigerating and Air Conditioning Engineers, Inc, Atlanta, EUA, 42 pp.

Consejo Mundial de Energía (2004). Comparison of energy systems using life cycle assessment [en línea]. http:// www.worldenergy.org/wp-content/uploads/2012/10/ PUB_Comparison_of_Energy_Systens_using_lifecycle_2004_WEC.pdf.

Consoli F., Allen D., Boustead I., Fava J., Franklin W., Jensen A. de Oude N., Parrish R., Perriman R., Postlethwaite D., Quay B., Séguin J. y Vigon B. (1993). Guidelines for life-cycle assessment: a 'code of practice' 1ra. ed. SETAC Press (Society of Environmental Toxicology and Chemistry). Pensacola, EUA, 73 pp.

Duffie J. A. y Beckman W. A. (2013). Solar engineering of thermal processes. 4ta. ed. J. Wiley and Sons, Hoboken, EUA, 910 pp.

Greening B. y Azapagic A. (2014) Domestic solar thermal water heating: a sustainable option for the UK ?. Renew. Energ. 63, 23-36.

DOI: $10.1016 /$ j.renene.2013.07.048.

Hammond G. y Jones C. (2011). Embodied carbon. The inventory of carbon and energy (ICE). University of Bath-BSRIA, Berkshire, Reino Unido, 136 pp.

Hang Y., Qu M. y Zhao F. (2012). Economic and environmental life cycle analysis of solar hot water systems 
in the United States. Energ. Buildings 45, 181-188. DOI: 10.1016/j.enbuild.2011.10.057.

Ihobe (2009). Análisis de ciclo de vida y huella de carbono: dos maneras de medir el impacto ambiental de un producto. Sociedad Pública de Gestión Ambiental, Gobierno Vasco, España, 37 pp.

IPCC (2013). Cambio climático 2013: Bases físicas. Resumen para responsables de políticas. Contribución del grupo de trabajo I al quinto informe de evaluación del grupo intergubernamental de expertos sobre el cambio climático. Grupo Intergubernamental de Expertos sobre el Cambio Climático, 34 pp.

Kalogirou S. (2009a). Solar energy engineering: processes and systems. Elsevier/Academic Press, Burlington, EUA, 760 pp.

Kalogirou S. (2009b). Thermal performance, economic and environmental life cycle analysis of thermosiphon solar water heaters. Sol. Energy 83, 39-48.

DOI: $10.1016 /$ j.solener.2008.06.005.

Koroneos C. J. y Nanaki E. A. (2012) Life cycle environmental impact assessment of a solar water heater. J. Clean. Prod. 37, 154-161.

DOI: $10.1016 /$ j.jclepro.2012.07.001.
Lamnatou C., Notton G., Chemisana D. y Cristofari C. (2014). Life cycle analysis of a building-integrated solar thermal collector, based on embodied energy and embodied carbon methodologies. Energ. Buildings 4 378-387. DOI: 10.1016/j.enbuild.2014.08.011.

NASA (2014). Surface meteorology and solar energy. A renewable energy resource web site. Atmospheric Science Data Center [en línea]. https://eosweb.larc.nasa. gov/cgi-bin/sse/grid.cgi.

OMM (2012). Declaración de la Organización Meteorológica Mundial sobre el estado del clima mundial en 2011, OMM-No. 1085, 24 pp.

REN 21 (2015). Renewables 2015 global status report. Annual Reporting on Renewables: Ten years of excellence, REN 21, $251 \mathrm{pp}$.

Rey-Martínez F. J., Velasco-Gómez E., Martín-Gil J., Navas Gracia L. M., Hernández Navarro S. (2008). Life cycle analysis of a thermal solar installation at a rural house in Valladolid (Spain). Environ. Eng. Sci. 25, 5, 713-724. DOI: 10.1089/ees.2007.0115. 